\title{
JUURNAL.RU
}

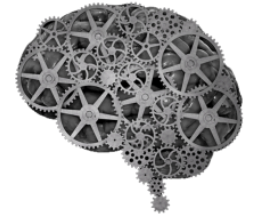

COMPANY GROUP "INTELLEKT"

Трифонова Т.А. Санкт-Петербургский национальный исследовательский университет информационных технологий, механики и оптики Санкт-Петербург, Россия

doi: 10.18411/1j2016-3-39

\section{Процедуры синтеза при обмене данными между автоматизированными системами при проектировании электронных средств}

Одним из важнейших направлений в развитии современного производства является автоматизация процесса проектирования и конструкторскотехнологической подготовки производства. При проектировании изделий современной электроники широко применяются различные автоматизированные системы (AC). Применение АC в современном производстве позволяет значительно снизить трудоемкость проектирования и планирования, тем самым сокращаются сроки проектирования изделий.

Автоматизированные системы классифицируются в зависимости от вида автоматизируемой деятельности. Так при конструировании электронных средств могут использоваться как системы автоматизации проектирования электроники (EDA - Electronic Design Automation), так и автоматизированные системы технологической подготовки производства (САM - Computer-aided Manufacturing) и системы механического проектирования (MCAD - Mechanical Computer-aided Design). Проектирование, к примеру, печатной платы электронного устройства включает в себя задание габаритов платы, количества слоев, трассировку или проектирование топологии, генерацию управляющих файлов для оборудования, изготовляющего фотошаблоны. Также проектирование может включать в себя создание трехмерной модели в системах механического проектирования, для последующей компоновки платы в 
электронный блок, испытания компьютерной модели платы в соответствующих системах и верификация модели. Различные этапы проектирования задействуют AC различного целевого назначения. Так для проектирования топологии печатной платы используют EDA-системы, для подготовки платы к производству и генерации управляющих файлов используют САМ-системы, для создания трехмерной модели используются как некоторые EDA-системы, так и MCAD-системы. Автоматизированное проектирование представляет собой информационный процесс, при котором происходит преобразование входной информации о проектируемом изделии, заданном в техническом задании, знаний инженера, данных о прототипах в выходную конструкторско-технологическую информацию. Такое преобразование данных фактически означает обмен данными о проекте в различных АС. Таким образом, обеспечение корректного обмена данными представляет собой основу успешного и эффективного автоматизированного проектирования. [1]

Сущность проектирования заключается в принятии проектных решений, обеспечивающих выполнение будущим объектом предъявляемых к нему требований. Синтез проектных решений - основа проектирования; от успешного выполнения процедуры синтеза в определяющей мере зависят потребительские свойства будущей продукции. [2]

Место процедур синтеза в процессе проектирования. Синтез делится, в свою очередь, на параметрический (или автоматизированный) и структурный. При структурном синтезе формируют структуру объекта, с которого и начинается проектирование. Дальнейшие действия производятся автоматизировано, т.е. производится параметрический синтез. Если параметрический синтез не приводит к успеху, то повторяют процедуры структурного синтеза, т. е. на очередных итерациях корректируют или перевыбирают структуру объекта. [2]

В системах автоматизированного проектирования используются как автоматизированные средства синтеза проектных решений, так и 
вспомогательные, выполняемые в интерактивном режиме инженеромконструктором. Задачи структурного синтеза являются наиболее трудноавтоматизируемыми, поэтому на многих этапах решающая роль отводится инженеру-конструктору и интерактивным средствам АС.

Преобразование данных между АC различного целевого назначения представляет собой один из этапов проектирования изделия, и соответственно включает в себя параметрический и структурный синтез.

\section{Оптимизация процесса трансляции включением структурного} синтеза. Проект печатной платы или интегральной схемы представляет собой совокупность топологических и геометрических математических моделей. Отражаемые в математической модели параметры оцениваются вектором параметров. При обмене данными о проектируемом изделии, из одной АС в другую передается вектор проектных параметров.

В АC представление модели на основе проектных параметров осуществляется за счет упорядоченной совокупности целевых функций.

$$
\gamma(\boldsymbol{X})=\left\{\begin{array}{l}
\gamma\left(\boldsymbol{X}_{1}\right)=F\left(\sum f_{i}\left(\boldsymbol{X}_{1}\right), \sum x_{j}\right) \\
\gamma\left(\boldsymbol{X}_{n}\right)=F\left(\sum f_{i}\left(\boldsymbol{X}_{n}\right), \sum x_{j}\right)
\end{array},\right.
$$

где $\boldsymbol{X}=\boldsymbol{X}_{1} \cup \boldsymbol{X}_{2} \cup \ldots \cup \boldsymbol{X}_{n}-$ совокупность всех топологических и геометрических параметров.

Процесс трансляции параметров модели в автоматизированном режиме показан на рис.1.

При эффективной трансляции вектора параметров все атрибуты объекта передаются без потерь, значения одних атрибутов не присваиваются другим и т. д. В системах различного целевого назначения количество параметров, необходимое для проектирования изделия может отличаться. Так в системах автоматизации проектирования электроники (EDA-системы) количество изменяемых атрибутов или параметров превышает количество параметров того же изделия в системах механического проектирования (MCAD-системы). 
Входные параметры модели $\mathrm{AC}$, из которой идет трансляция

$\gamma(\boldsymbol{X})$ - совокупность целевых функций
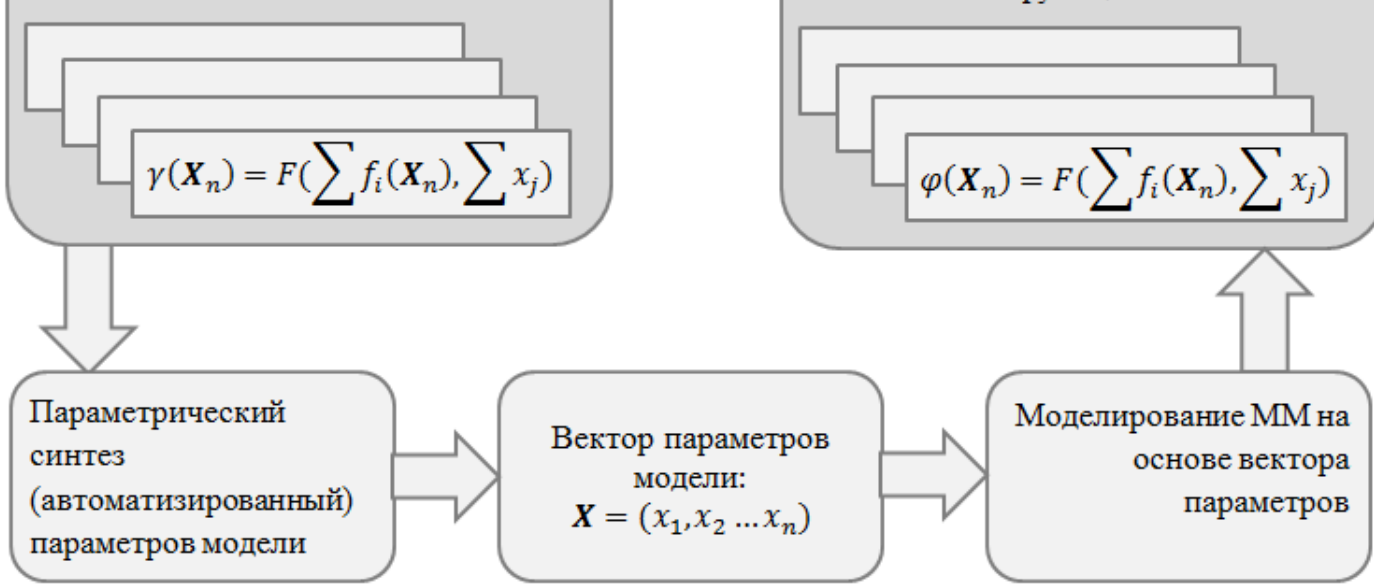

Рис. 1 - Трансляция параметров модели в автоматизированном режиме. $\varphi(\boldsymbol{X})$ - совокупность целевых функций

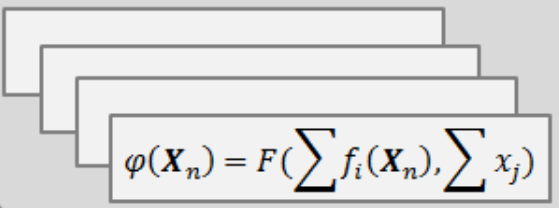

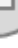 (n) pa}




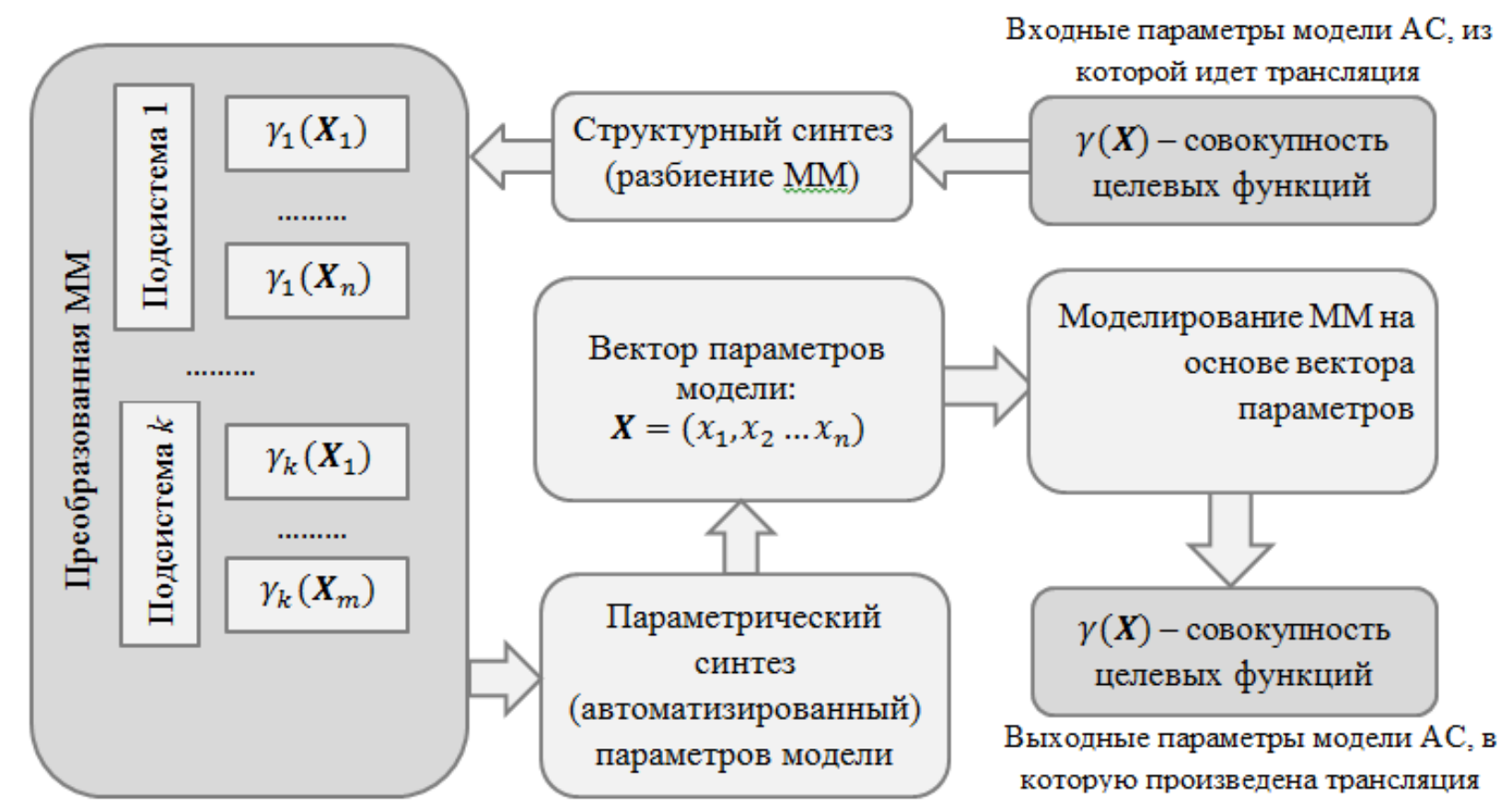

Рис. 2 -Трансляция параметров модели с использованием структурного синтеза.

Заключение. Оптимизация процессов трансляции данных является достаточно важной задачей, решение которой поспособствует увеличению эффективности проектирования. Процедуры структурного синтеза достаточно сложно поддаются автоматизации, особенно если задачи, решаемые в процессе данного синтеза, не определены. Определение общих подходов к постановке этих задач, а также решение данных задач с использованием эвристических методов, являются первыми шагами к автоматизированным способам решения. Принципы решения проблем в процессе структурного синтеза идут в основу принципов решения проблем в параметрическом или автоматизированном синтезе. 


\section{Литература:}

1 Рудинский И. Д., Технологии проектирования автоматизированных систем обработки информации и управления. - М.: Горячая линия - Телеком, 2011. - $304 \mathrm{c}$.

2 Норенков И.П., Основы автоматизированного проектирования. 2-е изд., перераб. и доп. - М.: Изд-во МГТУ им. Н.Э. Баумана, 2002. - 336 с.: ил.

3 Иванько А.Ф., Иванько М.А., Сидоренко В.Г., Фалк Г.Б. Автоматизация проектирования систем и средств управления. - М.: Изд-во МГУП, 2001. 148 с. [Электронный pecypc]: http://hi-edu.ru/e-books/xbook116/01/part003.htm 